\title{
Matériaux, travaux et ouvriers dans le Libro della fabbrica de Pratolino en 1575
}

Materials, constructions and workmens in the Libro della fabbrica of Pratolino in 1575

\section{Marco Calafati}

\section{OpenEdition}

\section{Journals}

Édition électronique

URL : http://journals.openedition.org/artefact/5402

DOI : 10.4000/artefact.5402

ISSN : 2606-9245

Éditeur :

Association Artefact. Techniques histoire et sciences humaines, Presses universitaires du Midi

Édition imprimée

Date de publication : 15 juillet 2020

Pagination : 73-88

ISBN : 978-2-8107-0691-4

ISSN : 2273-0753

\section{Référence électronique}

Marco Calafati, « Matériaux, travaux et ouvriers dans le Libro della fabbrica de Pratolino en 1575 »,

Artefact [En ligne], 12 | 2020, mis en ligne le 21 décembre 2020, consulté le 23 décembre 2020. URL: http://journals.openedition.org/artefact/5402 ; DOI : https://doi.org/10.4000/artefact.5402

\section{(c) $(1)$}

Artefact, Techniques, histoire et sciences humaines est mise à disposition selon les termes de la Licence Creative Commons Attribution - Pas d'Utilisation Commerciale - Pas de Modification 4.0 International. 


\section{Matériaux, travaux et ouvriers dans le Libro della fabbrica de Pratolino en 1575}

\section{Marco Calafati}

\section{Résumé}

Un manuscrit intitulé Libro delle bestie di Firenze e fabbrica di Pratolino (1575), conservé à l'Université de Pennsylvanie à Philadelphie, permet de connaître, à un moment central pour la réalisation des jardins, les matériaux, les travaux et les ouvriers employés sur le chantier de la Villa de Pratolino, au nord de Florence, commandé par François Ier de Medicis et conçu sous la direction de Bernardo Buontalenti de 1568 à 1586. La réalisation de la prodigieuse machine hydraulique de Pratolino est confiée aux ouvriers locaux, qui avaient appris les techniques d'ingénierie. On relève le nom du maître d'œuvre Davide Fortini, expert en génie hydraulique, actif en Toscane du milieu à la fin du xv $\left.\right|^{e}$ siècle et gendre de Niccolò Pericoli dit « il Tribolo » (vers 1500-1550). Le document de 1575 mentionne également des noms d'artisans, de maîtres maçons, de tailleurs de pierre, de charpentiers, ainsi que les différents matériaux qui arrivent dans la cour ; aussi, il cite le nom de « Tommaso di Gio. Parigi » représentant d’Alfonso Gondi.

\section{Mots-clés}

jardins, matériaux, travaux, ouvriers, Gondi, Pratolino, Renaissance

95 Marco Calafati, « Matériaux, travaux et ouvriers dans le Libro della fabbrica de Pratolino en $1575 »$, Artefact, 12, 2020, p. 73-88. 


\section{Materials, constructions and workmens in the Libro della fabbrica of Pratolino in 1575}

\section{Abstract}

A manuscript entitled Libro delle bestie di Firenze e fabbrica di Pratolino (1575), kept at the University of Pennsylvania in Philadelphia, allows us to know, at a central time for the creation of gardens, the materials, building work and workers employed on the construction site of the Villa of Pratolino, in the north of Florence. The villa was commissioned by Francesco I de' Medici and designed under the direction of Bernardo Buontalenti from 1568 to 1586. The production of Pratolino's prodigious hydraulic machine is entrusted to local workers, who had learned engineering techniques. We note the name of the master builder Davide Fortini, an expert in hydraulic engineering, active in Tuscany from the middle to the end of the 16th century, the son-in-law of Niccolò Pericoli known as "il Tribolo" (circa 1500-1550). The 1575 document also mentions the names of craftsmen, master masons, stonecutters, carpenters, as well as the various materials that arrived in the courtyard; it also mentions the name of "Tommaso di Gio. Parigi", representing Alfonso Gondi.

\section{Keywords}

gardens, materials, works, workmans, Gondi, Pratolino, Renaissance 
Élève-toi au-dessus de toute hauteur, descends au-dessous de toute profondeur ; rassemble en toi toutes les sensations des choses créées, de l'eau, du feu, du sec, de l'humide.

Suppose que tu es à la fois partout, sur la terre, dans la mer, dans le ciel [...] Comprends tout à la fois : les temps, les lieux, les choses, les qualités, les quantités. Corpus Hermeticum, Hermès Trismégiste ${ }^{1}$

L orsque Michel de Montaigne découvre Pratolino en 1580, il ne cache pas son émerveillement :

C 6 Le bâtiment y est méprisable à le voir de loin, mais de près il est très beau, mais non des plus beaux de notre France [...]. Il y a de miraculeux une grotte à plusieurs demeures et pièces : cette partie surpasse tout ce que nous ayons jamais vu ailleurs [...]. Il y a non seulement de la musique et harmonie qui se fait par le mouvement de l'eau, mais encore le mouvement de plusieurs statues, que l'eau ébranle et porte à divers actes, plusieurs animaux qui s'y plongent pour boire, et choses semblables [...]. La beauté et richesse de ce lieu ne se peut représenter par le menu².

La villa et le jardin de Pratolino (Fig. IX, cahier couleur) ont été créés dans un endroit inhospitalier, sans eau ni ressources naturelles en matériau de construction (pierres, sable et chaux). Le choix du lieu est déjà emblématique :

C 6 le duc de Florence y a bâti depuis douze ans, où il emploie tous ses cinq sens de nature pour l'embellir. Il semble qu'exprès il ait choisi une assiette incommode, stérile et montueuse, et voire sans fontaines, pour avoir cet honneur de les aller quérir à cinq milles de là, et son sable et chaux, à autres cinq milles. C'est un lieu, là, où il n'y a rien de plain ${ }^{3}$.

1. La citation se trouve (en italien) dans la dédicace du livre de Lensi Orlandi, 1978 (traduction en français de Louis Ménard).

2. Montaigne, 1974, p. 197-198.

3. Montaigne, 1974, p. 197. 
Pourtant, un jardin a été aménagé et aussitôt, il a été considéré comme une merveille, une création du génie humain, une œuvre exceptionnelle dans la forme, les effets et, surtout, pleine d'innovations. Pratolino était comme un laboratoire pour étudier, discuter et expérimenter. Les résultats ont été tels que le monde des eaux de Pratolino est devenu un archétype fondamental pour le jardin à travers l'Europe ${ }^{4}$.

\section{Modèles et prototypes}

Parmi les ingénieux artifices redécouverts au $\mathrm{XVI}^{\mathrm{e}}$ siècle pour capter des sources $^{5}$ afin d'embellir les résidences de campagnes des patriciens, au même titre que les allées étroites taillées en rideau, les clairières ou les labyrinthes, la grotte emplie de références au monde mythique des anciens, aux formules mystérieuses des alchimistes et la symbologie des savants, occupe une place à part. C'est le lieu idéal où l'union entre la Nature et l'Architecture se concrétise de la manière la plus intime et la plus originale, si bien que ces deux composantes s'interpénètrent et se confondent. Des réalisations hybrides et étranges nouent des liens entre l'artifice et la nature : des animaux pétrifiés ou vivants, des automates et des spectateurs, des plantes peintes et réelles, des sons naturels (comme le mouvement de l'eau) et ceux produits par le génie humain (comme le chant des oiseaux mécaniques), rivalisent pour susciter l'émerveillement des visiteurs. Un des plus illustres, Michel Eyquem, seigneur de Montaigne, dans son Voyage en Italie, célèbre les inventions des grottes des Médicis. Il fut enchanté dans le jardin de la Villa de Castello qu'il visita en 1580 par la " belle grotte, où il se voit toute sorte d'animaux représentés au naturel, rendant qui par bec, qui par l'aisle, qui par l'ongle ou l'oreille ou le naseau, l'eau de ces fontaines ${ }^{6} »$. La grotte est pleine de références allégoriques au rôle politique du grand-duc de Toscane, tel un nouvel Orphée, pacificateur et protecteur des arts, et son emplacement dans le jardin est une référence claire à son archétype, la grotte, conçue comme un refuge pour bêtes sauvages. Les animaux sont d'un marbre qui imite la couleur du pelage, les coquillages, les rocailles et les pierres qui ornent les murs sont en même temps des allu-

4. Grossoni 1999, 39, p. 17-39.

5. Selon Claudio Tolomei, Le lettere, cité dans Battisti, 1962. Sur les rapports entre nature et artifice, voir l'étude dirigée par Fagiolo, 1979.

6. Montaigne, 1974. 
sions aux merveilleuses œuvres de la nature et à l'extraordinaire habileté artistique et imitative de l'homme. Au compagnon mythique d'Eurydice qui, par son chant, envoûte les instincts des bêtes sauvages, fait écho l'écrivain, auteur de plusieurs traités d'agronomie, Agostino Del Riccio (14511598) qui compare la grotte, la maison des animaux, au palais, la maison des hommes :

Ma per cominciarci dal sontuoso palazzo che si deve fare per questa ragioni, come dicono i filosofi : la prima è per fuggire le gran piogge e grandini in guisa di animali razionali, ma quelli che non hanno ragione et discorso in loro rincorrono con natural estinto alle grotte, alle caverne e tane. Ma huomini giudiziosi per nostro commodo e salvezza fabbrichiamo case et palazzi stabili.

[Mais pour commencer le somptueux palais qu'il faut faire pour cette raison, comme disent les philosophes : le premier est de fuir les grandes pluies et la grêle sous l'apparence d'animaux rationnels, mais ceux qui n'ont pas de raison et de parole en eux courent avec l'extinction naturelle vers les grottes, les cavernes et les tanières.

Mais hommes de jugement, pour notre convenance et notre salut, construisons des maisons d'écurie et des palais.]

La Grotte des Animaux, comme on le sait, représente le point de départ d'un processus complexe qui, à Florence, exploite le thème des nymphées et des jeux d'eau souterrains. Dès lors, les grottes, constituant un contrepoint aux espaces en plein air, sont réinventées et recréées artificiellement ainsi même au-delà des Alpes, en France, soit au milieu des jardins dans des constructions originales, soit dans les sous-sols de la demeure. Après la Grotte des animaux de Castello, qui s'insère dans le thème du jardin où s'exprime le pouvoir de Cosme I $^{\text {er }}$ de Toscane sur les forces qui lui sont hostiles, nous passons à la Grande Grotte de Boboli (1583-1593). Ici, les trois salles conçues par Bernardo Buontalenti, entretiennent un rapport plus limité avec le jardin. La nature est recréée artificiellement dans les peintures des paysages alpins ou des pergolas, mais elle est surtout présente dans l'origine matérielle des éléments décoratifs : coquillages, cristaux, concrétions calcaires, spugne qui font référence au monde souterrain et qui produisent des figures et des images artistiques.

7. Del Riccio, 1981, p. 66. 
La différence entre les espaces naturels et les constructions artificielles, encore accentuée par leur distance physique réelle, s'estompa progressivement jusqu'à finalement disparaître. En effet, si la grotte est confinée dans les jardins à Castello et à Boboli, elle est située au cœur même de la villa à Pratolino, occupant le sous-sol du bâtiment où se trouvent également quelques pièces de service ${ }^{8}$. Le système complexe de salles, animées par des automates actionnés par des dispositifs hydrauliques sophistiqués, est l'un des premiers exemples de grotte souterraine communiquant avec la résidence, semblables à celles de la Villa d'Este à Tivoli : l'entrée principale donne sur le jardin, mais un passage secondaire permet d'accéder à la cuisine secrète, à la confiserie et à la cave à vin où les domestiques, en plus de s'occuper des services quotidiens, peuvent, si nécessaire, organiser des banquets au sous-sol. La grotte, point d'appui dans lequel les quatre éléments, l'eau, la terre, l'air et le feu, sont unis, prend une valeur symbolique en tant qu'origine de l'homme et source de sa renaissance morale. Il n'en subsiste que quelques vestiges (Fig. $\mathbf{X}$ et $\mathbf{X I}$, cahier couleur).

\section{Une redécouverte récente}

Pratolino était au cœur de la réflexion de Battisti lorsqu'il entreprit une relecture complète du Cinquecento dans son grand livre L'Antirinascimento paru en 1962 et dans lequel la nature dans les arts occupait une place importante ${ }^{9}$. En 1967, dans son étude sur François I ${ }^{\text {er }}$ de Médicis, Luciano Berti a proposé une interprétation de Pratolino au regard de la personnalité du grand-duc. Peu après, Detlef Heikamp proposait une reconstitution de Pratolino et soulignait les nombreuses innovations dont le jardin avait fait preuve, suivi en cela par Franco Borsi. Mais c'est seulement en 1979 que parut la monographie de Luigi Zangheri, étude qui offrit enfin une vue d'ensemble et un historique détaillé de la villa en s'appuyant sur une riche documentation d'archives inédites ${ }^{10}$. Par la suite, plusieurs colloques et des chantiers de restauration ont été coordonnés par Marco Dezzi Bardeschi et Alessandro Vezzosi ${ }^{11}$. Par ailleurs, Cristina Acidini Luchinat, Herbert

8. Zangheri, 1987.

9. Battisti, 1989, vol. I, p. 268-286.

10. Berti, 1967, p. 85-107 ; Heikamp, 1969 ; Borsi, 1993, p. 205-211 ; Zangheri, 1979.

11. Dezzi Bardeschi, Vezzosi 1985. 
Keutner ou Mariachiara Pozzana, aux côtés de Luigi Zangheri et de Detlef Heikamp, ont poursuivi des recherches sur l'histoire du jardin ${ }^{12}$.

D’une manière générale, la place des jardins de Pratolino dans l'historiographie des jardins a été marquée par les travaux de Marcello Fagiolo ${ }^{13}$, et par un renouveau des études sur la Toscane médicéenne promu par les grandes expositions de Florence en $1980^{14}$. Cette question a été reprise par Fausto Testa et dans une mise au point, tentée par Lionello Puppi, autour de la notion d'illusion. Ensuite, Philippe Morel a mis en lumière les automates et les grottes dans la culture de la Renaissance ${ }^{15}$ tandis que Claudia Lazzaro a souligné que la conception d'ensemble du jardin était une représentation de la nature ${ }^{16}$. Quant à la thèse de doctorat de Hervé Brunon consacrée à Pratolino ${ }^{17}$, elle aborde l'art des jardins dans l'Italie de la seconde moitié $\mathrm{du} \mathrm{XVI}{ }^{e}$ siècle comme une expression des rapports entre l'homme et la nature en recourant à la notion d'imaginaire. Cette approche a été reprise récemment pour montrer que Pratolino est une exploitation politique des représentations culturelles de la nature.

L'ensemble de ces recherches a donc permis de rassembler une vaste documentation et de tracer plusieurs lignes d'interprétations. L'intérêt du Libro della fabbrica de Pratolino est de porter un regard différent sur les jardins avec une documentation riche pour l'histoire technique, l'ingénierie et l'hydraulique.

\section{Travaux et ouvriers}

On doit à François I $^{\text {er }}$ de Medicis (1541-1587), qui a commandé la villa de Pratolino et ses rapports avec les princes de l'époque, la fortune du jardin avec des nymphées anthropomorphes, tandis que des livres, comme celui de Francesco De Vieri, Delle meravigliose opere di Pratolino et d'Amore (1586) qui analyse le jardin comme un merveilleux lieu de rêve et d'enchantement,

12. Acidini, 2008, p. 137-153, Zangheri, 1987, 1991 et 1995 ; Heikamp, 1994.

13. Fagiolo, 1979 et 1980.

14. Ragionieri, 1981 ; Borsi 1980.

15. Testa, 1991 ; Puppi, 1991 ; Morel, 1987 et 1990.

16. Lazzaro, 1990.

17. Brunon, 2001 ; Brunon, Mosser 2014. 
sont diffusés dans toute l'Europe ${ }^{18}$. Après l'acquisition de la propriété de Pratolino par le grand-duc François I ${ }^{\text {er }}$ en 1568, les travaux débutèrent avec un projet de Bernardo Buontalenti et durèrent jusqu'en 1586, après quoi il y eut encore une série de remembrements des terres agricoles autour de la villa.

Un manuscrit original intitulé Libro delle bestie di Firenze e fabbrica di Pratolino (1575), conservé à l'Université de Pennsylvanie à Philadelphie ${ }^{19}$, permet de connaître, à un moment central, les commandes de matériaux, les travaux réalisés et les ouvriers employés sur le chantier. Sur ce livre de compte, on relève les noms du maître d'œuvre Davide Fortini et de "Niccolò son fils ", expert en génie hydraulique, actif en Toscane du milieu à la fin du xvie siècle. Davide Fortini était le fils de Raffaello Fortini et le gendre de Niccolò Pericoli dit «il Tribolo » (vers 1500-1550), dont il a épousé la fille aînée Dianora. Il avait commencé sa carrière en travaillant sous la direction de son beau-père. Avec Tribolo, Fortini collabore, à partir de 1550 environ, à la construction de la Villa de Castello pour Cosme ${ }^{\text {er }}$ de Médicis, où il continue à être employé même après la mort de Tribolo en septembre 1550, gérant le site jusqu'en 1554, date à laquelle Giogio Vasari prend la relève. En 1549, il est engagé dans les écuries de la villa de Poggio a Caiano, où il prend des décisions sur le choix des matériaux et les méthodes de construction. Vers 1550, il fait partie de la Magistratura dei Capitani en tant que maître d'œuvre, exerçant principalement la fonction de défenseur des intérêts ducaux dans les procès ${ }^{20}$. En 1570, Fortini débute une période de travail intense pour le compte de la Magistratura des rivières puis, en 1575, pour le compte du cardinal Ferdinand de Médicis, il travaille sur le projet de drainage du Val di Chiana. Durant cette période, il a été employé souvent dans les villas des Médicis. Si, à partir de 1566, il dirigea les travaux du jardin de Petraia ${ }^{21}$, toujours en tant que maître d'œuvre, il fut aussi actif dans les travaux de la villa de Pratolino conçue

18. De Vieri, 1587, p. 34 et 64 . De Vieri propose une lecture dans les tons moralisateurs de Pratolino. Voir dans Scritti d'arte del Cinquecento, édité par P. Barocchi, Milan-Naples 1971, III, p. 3408. À ce propos, Brunon, 2007 ; Brunon et Mosser, 2014 ; Brunon, 2017.

19. Philadelphie, Université de Pennsylvanie, Codex 1551. J'ai consulté et photographié le manuscrit à l'automne 2010 dans les collections spéciales de la bibliothèque de l'Université de Pennsylvanie où j'ai étudié les archives des Gondi grâce à une bourse de l'Institut italien des sciences humaines (Istituto Italiano di Scienze Umane, Palazzo Strozzi, Florence).

20. Ferretti, 2001; Ferretti, 2016.

21. Acidini, 1992, p. 164. 
par Bernardo Buontalenti. Il a aussi travaillé dans le jardin de la villa de Poggio a Caiano, sur le lac de la Villa della Màgia et à la construction de la villa de Coltano près de Pise $^{22}$.

Dans le Libro della fabbrica de Pratolino de 1575, sont également mentionnés les peintres Giovanni dit Lo Spagna, Francesco dello Sciorina, et Baccio di Michele pittore, probablement à identifier avec Tosini Baccio di Michele di Jacopo di Rodolfo, inscrits parmi les membres de l'Académie de l'Art du Dessin. Lorenzo Vaiani, également appelé la Sciorina (1540-1598), appartient au cercle des peintres maniéristes actifs à Florence dans la seconde moitié du Xvi siècle. Fils de Filippo, surnommé Sciorina, Lorenzo Vaiani fréquentait l'atelier de Bronzino et fut l'élève d'Alessandro Allori. En 1563, il entre à l'Académie du dessin de Florence et participe à de grands projets tels que les Esequie del divino Michelangelo ou le second mariage du prince François $\mathrm{I}^{\mathrm{er}}$ de Médicis, événements marquants de l'histoire artistique florentine de la seconde moitié du XVI siècle.

La réalisation de la prodigieuse machine hydraulique de Pratolino est confiée aux ouvriers locaux, qui avaient appris les techniques d'ingénierie grâce à des collaborations avec des ouvriers compétents, ou bien grâce à l'apprentissage auprès de maîtres expérimentés, en tout cas ils étaient en mesure de réaliser des ouvrages de haute technicité. En plus des noms des artisans, des maitres maçons, des tailleurs de pierre, des charpentiers, ce document de 1575 indique les matériaux livrés pour le chantier. Les comptes mentionnent ainsi de grandes quantités de sable, des pierres, des planches de bois, des briques et des tuiles, ainsi que des fournitures de plomb livrées à «Antonio di Bernardo et Pasquino son apprenti ».

Le Libro della fabbrica de Pratolino rapporte également le nom d'Alfonso Gondi comme administrateur des comptes des travaux et celui de «Tommaso di Gio. Parigi » qui le représentait en son absence (Fig. 1) ${ }^{23}$. On peut donc supposer une connaissance directe et approfondie de Pratolino par les membres de la famille Gondi, dont certains se sont installés à Lyon au début du xvi ${ }^{e}$ siècle comme marchands-banquiers, puis à Paris comme

22. Fara, 1988, p. 200.

23. Université de Pennsylvanie, Codex 1551, fol. $14 \mathrm{r}$ : «Alfonso Gondi di contro de havere a dì 10 di settembre trentaquattro di moneta, recò Tommaso Parigi, conto 138 "; fol. 20r (voir en annexe). À cette époque, un certain Alfonso Gondi (1522-1574) dirige la Maison de la Reine de Navarre, en France. Salviati, 1975, p. 116 (lettre de Salviati à Galli, Lyon, 29 novembre 1574). 
ambassadeurs et diplomates dans la seconde moitié du siècle. Cette double appartenance des Gondi à l'État florentin et au royaume de France se reflétait dans les choix artistiques et architecturaux de leurs demeures.

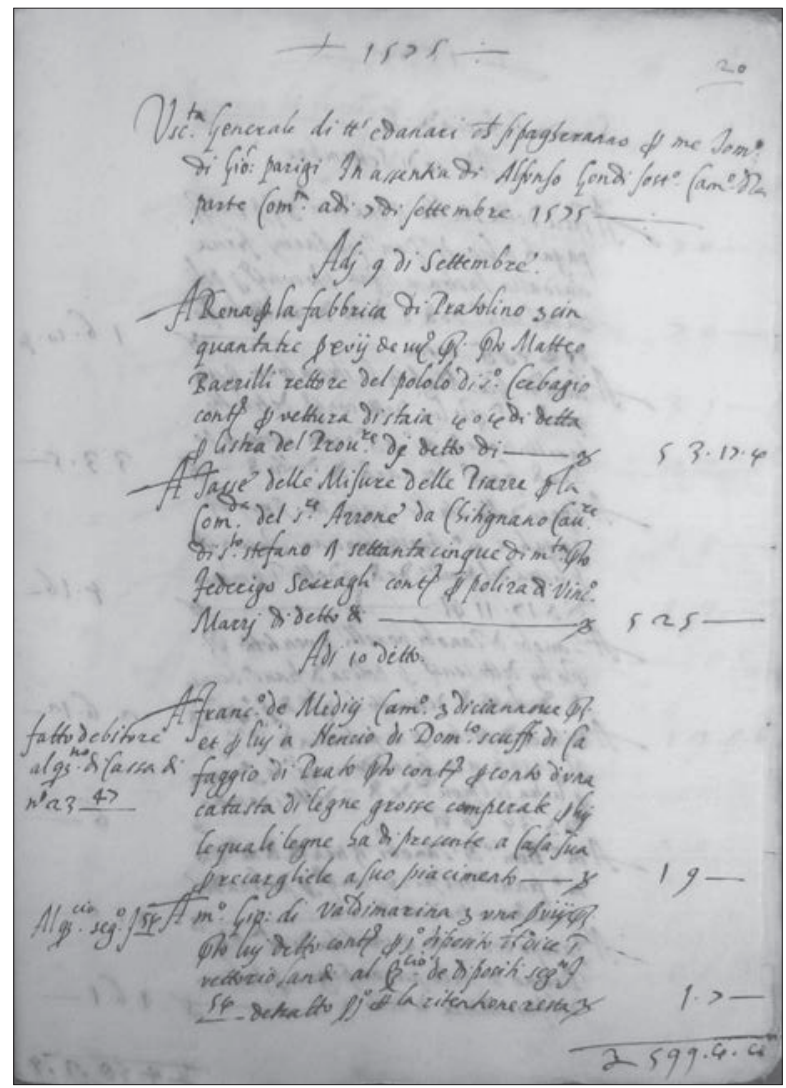

Fig. 1. - Libro delle bestie di Firenze e fabbrica di Pratolino, 1575

Université de Pennsylvanie, Philadelphie, Ms. Codex 1551, fol. 20r).

Cliché Marco Calafati (transcription en annexe)

De même, sur le modèle de Pratolino, de nombreuses résidences florentines ou celles de Florentins à l'étranger, proposent un nouveau rapport entre leur demeure et la grotte artificielle. L'eau, prépondérante dans les villas des Médicis, apparaît alors en abondance dans les pépinières, elle traverse des tunnels pour animer des cavernes secrètes de la demeure, les nymphées, enfin elle s'infiltre dans le sol du jardin de fleurs et de fruits qui est source de vie. Le système hydraulique réalisé utilise des techniques déjà 
largement éprouvées et illustrées par les traités, ce qui reflète également l'expertise hydraulique réelle de l'école toscane d'ingénieurs. L'usage somptuaire de l'eau témoigne de la primauté du génie hydraulique et célèbre l'eau comme un matériau rare, précieux et extrêmement riche.

Déjà dans les Relazioni di Francia de 1577, l’ambassadeur vénitien Girolamo Lippomano inclut Noisy parmi les magnifiques constructions autour de Paris

CC dove si veggono archi, acquidotti, statue, giardini, parchi, peschiere, e tutte quelle commodità in fine, che si ricercano a edificii regii

\ $C$ [où l'on peut voir des arcs, des aqueducs, des statues, des jardins, des parcs, des étangs à poissons, et finalement toutes ces commodités qui sont recherchées dans les bâtiments royaux $]^{24}$

Fig. 2. - Lettre de l'ambassadeur extraordinaire en France, Andrea Albertani, au grandduc de Toscane, François fer $^{-}$ de Médicis, 1582

Florence, Archives d'État, Mediceo del Principato, 4611, fol. 113 bis.

Cliché Marco Calafati

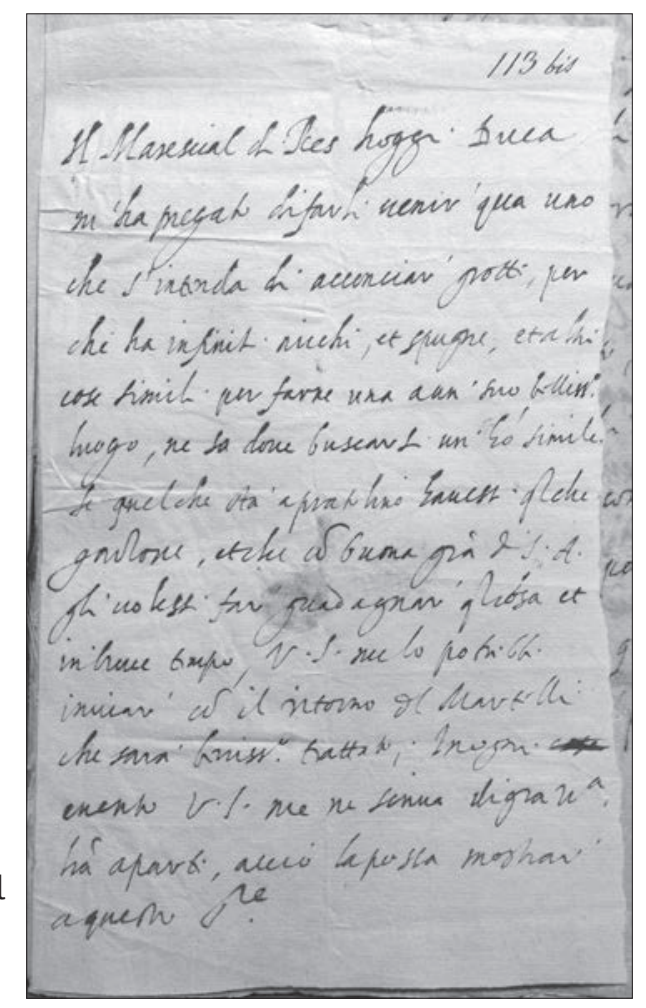

24. Relations..., 1838, p. 488-490. Voir aussi Lippomano, 1579. 
Dans une lettre de 1582 à François Ir de Médicis (Fig. 2), Albertani précise que

Se qualche costà a Pratolino havessi qualche garzone, et che con buona grazia di Sua Altezza gli volessi far guadagnare qualcosa et in breve tempo, Vostra Signoria me lo potrebbe inviare con il ritorno del Martelli.

[Si vous avez un garçon à Pratolino qui, avec l'approbation de Son Altesse, est d'accord pour gagner quelque chose en peu de temps, Votre Éminence pourrait me l'envoyer avec le retour de Martelli] ${ }^{25}$

Il s'agit peut-être de Ugolino Martelli (1519-1592), évêque de Glandèves en Provence entre 1572 et 1591, qui fit de nombreux voyages avec l'évêque Strozzi dans la suite de la cour de France, alors dominée par la personnalité de Catherine de Médicis et des Italiens de son entourage ${ }^{26}$. La réponse de François I ${ }^{e r}$ n'est pas connue, mais il est certain que Pratolino était le point de départ de la formation des ingénieurs hydrauliques parmi lesquels se distinguèrent ensuite les Francini.

Ce livre de compte est donc un document d'archives exceptionnel tant il est rare de disposer, pour les grottes artificielles de la Renaissance, de sources écrites et plus particulièrement d'actes comptables ou administratifs. Il vient en outre compenser la disparition de la plupart des constructions et des aménagements réalisés à Pratolino, que nous connaissons d'abord grâce à des sources graphiques. Enfin, ce manuscrit apporte des indications précises sur l'organisation d'un chantier et fournit des données techniques et financières qu'il est extrêmement rare de posséder pour ce genre d'ouvrage. Il apporte ainsi de nouvelles données sur les échanges de savoirs et les circulations des artisans à la fin du XVI ${ }^{\mathrm{e}}$ siècle.

25. Florence, Archives d'État, Mediceo del Principato, 1581-1583; 4611, fol. 113 bis.

26. Les lettres entre Martelli et Pier Vettori sont la source principale pour suivre les événements d'Ugolino en France dans les années entre les règnes d'Henri II et d'Henri III. Bramanti, 2008. Brock, 2011. 


\section{Annexe}

Transcription du manuscrit reproduit figure 1

fol. $20 r$

1575

Usc[i]ta generale di t[ut]ti e danari che si pagheranno per me Tom[maso] di Gio[vanni] Parigi in assentia di Alfonso Gondi sost[ituit]o com. ${ }^{\circ}$ dalla parte com.ta a dì 7 di settembre 1575 .

A dj 9 di settembre

A Rena per la fabbricha di Pratolino lire cinquantatre scudi xvii denari iiij [...] per lo Matteo Barzilli rettore del popolo di S[ant]o Cerbagio cont[anti] scudi per vettura di staia 4 o 4 di detta per listra del Prov[vedito] re dj detto dì 53.17 .4

A tasse delle Misure delle Piazze per la Com[men]da del S[enator]e Arrone da Chitignano Cav[alie]re di S[an]to Stefano scudi settantacinque di m[one]ta per lo Federigo Serragli cont[anti] per poliza di Vinc[enz]o Marzi di detto dì,

lire $525-$

A dì 10 detto

Fatto debitore al quaderno di Cassa di n. 23 - 47 A Franc[esc]o de Medicj $\mathrm{Cam}^{\circ}$ lire diciannove $[. .$.$] et per lui a Nencio di Dom[enic]o Scuffi di$ Cafaggio di Prato quello cont[anti] per conto di una catasta di legne grosse comperate per lui le quali legne ha di presente a casa sua per recargliele a suo piacimento, lire 19 -

Al quadernaccio seg[nat]o 54. A m[aestr]o Gio[vanni] di Valdimarina lire una, scudi otto quello lui detto conto per $1^{\circ}$ diposito che vice $\mathrm{T}$ [in] vettorio Gondi al b[an]cio de dipositi seg[na] to F 54 detratto $1^{\circ}$ per la ritentione resta lire 1.7 -

lire 599.4.4- 


\section{Sources}

Florence, Archives d'État [Archivio di Stato di Firenze], Mediceo del Principato, 1581-1583, n 4611, fol. 113 bis.

Philadelphie, Université de Pennsylvanie, Ms. Codex 1551, "Libro delle bestie di Firenze e fabbrica di Pratolino ", https://dla.library.upenn.edu/dla/medren/ pageturn.html?id=MEDREN_9945394023503681\&fq

[Michel de Montaigne], Journal du Voyage de Michel de Montaigne en Italie, par la Suisse et par l'Allemagne en 1580 et 1581, éd. Meusnier de Querlon, Rome et Paris, Le Jay, 1774.

Del Ricio Agostino, Del giardino di un re, éd. D. Heikamp, dans Il giardino storico italiano, actes du colloque de Sienne - San Quirico d'Orci, 6-8 octobre 1978, Florence, 1981.

De VIeRI Francesco, Delle meravigliose opere di Pratolino et d'Amore, Florence 1587.

Correspondance du nonce en France Antonio Maria Salviati (1572-1578), t. II (1574-1578), éd. P. Hurtubise et R. Toupin, Rome, 1975.

Relations des ambassadeurs vénitiens sur les affaires de France au XVI siècle, recueillies et traduites par M.N. Tommaseo, Paris, 1838, t. II, Paris 1838, p. 488-490.

Lippomano Girolamo, Relazione di Francia. 1579, in Relazioni di ambasciatori veneti al Senato: tratte dalle migliori edizioni disponibili e ordinate cronologicamente, L. Firpo (dir.), t. V, (France, 1492-1600), Turin, 1978, p. 775-812.

\section{Bibliographie}

Acidini Cristina, La ville e i giardini di Castello e Petraia a Firenze, Florence, Cassa di Risparmio di San Miniato, 1992.

Acidini Cristina, "Le cornici delle acque ", dans La fonte delle fonti, Alinea, Florence, 1985, p. 7- 12.

Acidini Cristina, "L'Appennino dal modello all'opera compiuta ", dans Risveglio di un colosso, Alinari, Florence, 1988, p. 13-21.

Acidini Cristina, "Le miniere della terra, i giacimenti del mare. Per un catalogo dei materiali dentro il Colosso ", dans L’Appennino del Giambologna, Alinea, Florence, 1990, p. 95-98.

Acidini Cristina, I giochi delle acque e gli stupendi artifici nel parco di Pratolino, par Simonetta Merendoni et Luigi Ulivieri, Marsilio, Venise, 2008.

Batтisti Eugenio, L'Antirinascimento, Milan, Feltrinelli, 1962.

Borsi Franco, Il potere e lo spazio, la scena del principe, Electa Editrice, Florence, 1980. Borsi Franco, L'architettura del principe, Florence, Giunti Martello, 1980.

Borsi Franco, Firenze del Cinquecento, Rome, Editalia, (1 re éd. 1974), 1993. 
Bramanti Vanni, "Ugolino Martelli », dans Dizionario Biografico degli Italiani, $n^{\circ} 71$, Rome, 2008, p. 64-67.

Brock Maurice, "Le "Portrait d'Ugolino Martelli" par Bronzino, un "Homère" florentin..." , dans Capodieci L., Homère à la Renaissance : mythe et transfigurations, Paris, Ph. Ford, 2011, p. 323-344.

Brunon Hervé, Pratolino : art des jardins et imaginaire de la nature dans l'Italie de la seconde moitié du XVI siècle (sous la direction du professeur Daniel Rabreau), université Paris I Panthéon-Sorbonne, 2001 (édition numérique 2008, HAL archives-ouvertes.fr).

BRUnOn Hervé, «Une scintillante pénombre : vingt-cinq ans de recherches sur les grottes artificielles en Europe à la Renaissance ", Perspective, n 2, 2007, p. 341-376.

Brunon Hervé et Mosser Monique, Limaginaire des grottes dans les jardins européens, Paris, 2014.

Brunon Hervé, "Quelques topiques dans l'imaginaire des grottes en Europe ", dans Zangheri, Luigi, Ville e Giardini Medicei in Tuscany and their influence in the art of gardens, Florence, 2017, p. 13-36.

Dezzi Bardeschi Marco, Vezzosi Alessandro, Il ritorno di Pan, Pratolino, Laboratorio di Maraviglie, Provincia di Firenze, Firenze, 1985.

FaGiolo Marcello (dir.), Natura e artificio, Rome, Officia, 1979.

FAGIOLO Marcello, La città effimera e l'universo artificiale del giardino: la Firenze dei Medici e l'Italia del '500, Officina, 1980.

FARA Amelio, Bernardo Buontalenti: l'architettura, la guerra e l'elemento geometrico, Gênes, Sagep, 1988.

Ferrettr Emanuela, "Maestro David Fortini from Tribolo to Buontalenti, a career in the shadow of the greats ", dans Pieri E. et Zangheri Luigi, Niccolò detto il Tribolo, tra arte, architettura e paesaggio, Comune di Poggio a Caiano, Florence, Signa, 2001, p. 73-85.

Ferretti Emanuela, Acquedotti e fontane del Rinascimento in Toscana, acqua, architettura e città al tempo di Cosimo I dei Medici, Florence, Leo S. Olschki, 2016.

Grossoni Paolo, "Formalismo e naturalità nel parco di Pratolino ", dans Rivista di Storia dell'Agricoltura, 39, 1999, p. 17-39.

Heikamp Detlef, "Les Merveilles de Pratolino ", L'Eil, n 171, mars 1969, p. 16-27 et 74-75.

Heikamp Detlef, «Pratolino nei suoi giorni splendidi ", Antichità viva, vol. VIII, $\mathrm{n}^{\circ} 2,1969$, p. 14-34.

Lazzaro Claudia, The Italian Renaissance Garden, Yale University Press, New Haven, CT, 1990.

Lensi Orlandi Giulio, Cosimo et Francesco de Medici alchimisti, Florence, Cardini, 1978. 
Morel Philippe, "Osservazioni sugli automi nel loro rapporto con le grotte, alla fine del Rinascimento ", dans Arte delle grotte, 1987, p. 59-64 (repris dans Morel, 1998, chapitre IV, p. 107-121).

Morel Philippe, "Secret, hermétisme et pouvoir d'État dans l'art médicéen à la fin du Xvi e siècle ", dans Dujardin Philippe (dir.), Le Secret, Lyon, Presses universitaires de Lyon, 1987, p. 31-62.

Morel Philippe, Les Grottes maniéristes en Italie au XVI siècle : théâtre et alchimie de la nature, Macula, Paris, 1998.

Morel Philippe, "La théâtralisation de l'alchimie de la nature. Les grottes artificielles et la culture scientifique à Florence à la fin du XVI siècle ", dans Symboles de la Renaissance, Paris, Presses de l'École Normale Supérieure, vol. III, 1990, p. 154-183 (repris dans Morel, 1998, chapitres I-III, p. 5-106).

Peroni Chiara, "L'ozio e la frescura : ninfei sotterranei a Firenze tra XVI e XVII secolo ", dans Dalu Jones, Il teatro delle acque, Rome, 1992, p. 90-104.

Puppi Lionello, "Nature, artifice et illusion. Thèmes et problèmes du jardin italien au Xvie siècle ", dans Histoire des jardins, 1991, p. 43-54.

Ragionieri Giovanna, Il giardino storico italiano, Regione Toscana, Giunta Regionale, Olschki, Florence, 1981.

Testa Fausto, Spazio e allegoria nel giardino manierista. Problemi di estetica, Florence, La Nuova Italia, coll. « Pubblicazioni della Facoltà di lettere e filosofia dell'Università di Pavia. Dipartimento di Scienza della letteratura e dell'Arte Medioevale e Moderna ", 1991.

Vezzosi Alessandro, «Pratolino d'avanguardie : Fonti d'ingegni stupori e controveleni ", dans La fonte delle fonti, Alinea, Florence, 1985, p. 49-67.

Zangheri Luigi, Pratolino, il giardino delle meraviglie, Florence, Gonelli, 2 vol., 1979 (rééd. 1987).

\section{L'auteur}

Marco Calafati enseigne l'Histoire de l'art à l'Université de Florence au Centro di Cultura per Stranieri. Il a soutenu une thèse de post-doctorat en Histoire de l'art de la Renaissance (École pratique des hautes études, Sorbonne-Paris), sur le mécénat artistique de la famille Gondi entre l'Italie et la France ou xvle siècle. En poursuivant son travail sur l'œuvre de Bartolomeo Ammannati, élève de MichelAnge, commencé par un mémoire de Master 2 puis dans une thèse, soutenue en 2008 (Dipartimento di Storia dell'Architettura e della Città dell'Università di Firenze), il a concentré ses recherches sur l'architecture et les spécificités des palais de la Renaissance à Florence. Il a notamment publié une monographie sur les palais de Florence : Bartolomeo Ammannati, I palazzi Grifoni e Giufoni. La nuova architectura dei palazzi fiorentini del secondo Cinquecente, Florence, Leo S. Olschki, 2011. Contact : marco.calafati@unifi.it 